\title{
Application of longitudinal analysis to the analysis of height increment of pine stands - simulated data
}

\author{
Bogna Zawieja \\ Department of Mathematical and Statistical Methods, Poznań University of Life Sciences, \\ Wojska Polskiego 28, 60-637 Poznań, Poland, bogna13@up.poznan.pl
}

\section{SUMMARY}

\begin{abstract}
One of the most important characteristics in determining the dendrometrical properties of a stand is the annual height increment of the trees. On the basis of these increments, natural phases of the trees' life are defined. In the present study the data concern the height increments of the main trunk of 25-year-old Scots pine, as well as simulated data. Our research deals with the application of longitudinal data analysis. This analysis is usually used when measurements are taken at the same treatments at different time points. The calculations performed provide answers to the question of which annual height increments differ.
\end{abstract}

Key words: contrast, height increment, longitudinal data analysis, Scots pine (Pinus sylvestris).

\section{Introduction}

In research conducted in pine forests, the height of trees is among the values measured. Since in successive years the measurements are performed on the same experimental units (trees), this data may not be regarded as independent. In experiments with repeated measurements the analysis of variance cannot be used to test the hypothesis of equality of means for years. In this situation profile analysis (also called longitudinal analysis) is used, in which two kinds of experimental factors are considered. Levels of some factors are tested on different experimental units, whereas levels of the other factors are examined on the same experimental units. In the trial analyzed in this paper, the first factor is associated with the division of the tested trees into Kraft classes (Assmann, 
1968), while the other is the measurement of tree heights in successive years. In work by Graczyk et al. (2010) longitudinal analysis was used for the one-factor experiment, i.e. the successive years.

The second factor, Kraft classes, combines the social position of the tree in the stand with the degree of its crown formation. Particular units are allocated to appropriate classes on the basis of visual assessment of the tree and its neighborhoods (Kaźmierczak and Zawieja, 2008).

\section{Data}

Data was simulated on the basis of real height measurements of 24-year-old pines. A total of 25 trees were selected, following the methodology developed by Draudt (Lemke, 1971). Observations of height were made for seven years. Before being cut down the trees were classified into Kraft classes (Assmann, 1968). Trees belonging to the same class are grouped with uniform growth energy. The considered 25 trees belonged to four different classes: first (I) predominant trees, markedly higher than surrounding trees, conspicuously protruding over the closed canopy, with very strongly developed crowns; second (II) dominant trees, forming the primary line of the closed canopy, with relatively well-developed crowns; third (III) co-dominant trees, slightly lower than dominant trees, with markedly less developed crowns, narrowed sideways; and fourth (IV) intermediate trees, markedly lower, with poorly developed, narrowed crowns, extending only in one direction.

Based on this experimental data, the simulated data was generated. Namely, separately for each Kraft class, the vector of means and covariance matrices among years were determined. Next, using the Statistical Analysis Software SAS, multivariate data (where the dimensions were the years) was generated from a normal distribution with a given vector of means and given covariance matrix. Consequently the simulated data, for each class, was independent for different experimental units (trees) and was dependent on the inside of the experimental unit (the height of a given tree in subsequent years). 


\section{Method}

In the presented experiment the data is dependent on time, and the measurements are repeated on the same experimental units. In this situation, longitudinal analysis should be used to verify hypotheses about factors (Kraft classes, years and trees).

In this analysis the influence of the $p$ commensurable responses on the $n$ independent objects which are grouped adequately according to $k$ experimental conditions is determined. The commensurable responses are the measurements made in consecutive years, in other words the time points. Social positions (Kraft classes) are the changing experimental conditions. In each of these classes the same number of trees is located (these trees are independent objects). Therefore there are observed $k$ groups consisting of $n$ objects at $p$ time points. The applied model can be described as follows:

$$
y_{i j h}=\xi_{j h}+e_{i j h}
$$

where $y_{i j h}$ is the observation concerning the measurement of the $i$ th object from the $j$ th group at the $h$ th time point, $\xi_{j h}$ is the influence of the $h$ th time point on the $j$ th group, and $e_{i j h}$ is the measurement error, $i=1,2, \ldots, n, j=1,2, \ldots, k$, $h=1,2, \ldots, p$. In the above model it is assumed that the error vectors $e_{i j}^{\prime}=\left[e_{i j 1}, \ldots, e_{i j p}\right]$ are independent, and have the multivariate normal distribution with expectation equal to zero and unknown nonsingular covariance matrix $\Sigma$, which is identical for each $i=1,2, \ldots, n, j=1,2, \ldots, k$, and is a matrix of full column rank. The model (1) can be also written in the matrix form $\mathbf{Y}=\mathbf{X} \boldsymbol{\xi}+\mathbf{e}$, where $\mathbf{Y}$ is the observation matrix. The matrix rows $\mathbf{Y}$ are in the form $y_{i j}=\left[y_{i j 1}, \ldots, y_{i j p}\right]$, for observation $i=1,2, \ldots, n$ and group $j=1,2, \ldots, k$.

The design matrix $\mathbf{X}$ is a matrix built of $k$ blocks of $n$ rows and $k$ columns each. In $j$ th block the $j$ th column is a column of ones, and the other columns are zero; hence the $j$ th block is: 


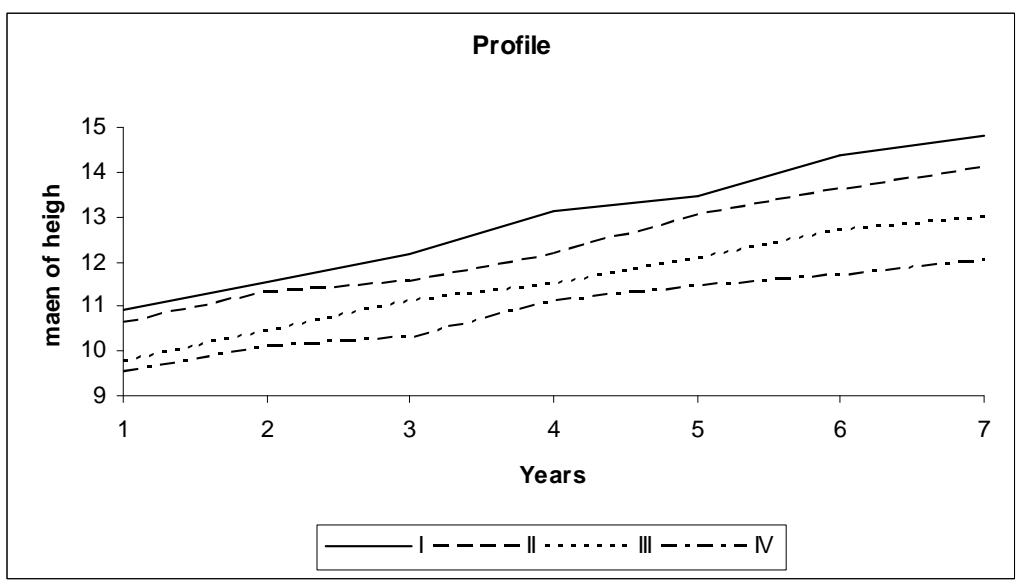

Figure 1. The profile of all generated tree heights from four Kraft classes

$$
\left[\begin{array}{ccccc}
0 & \ldots & 1 & \ldots & 0 \\
0 & \ldots & 1 & \ldots & 0 \\
\vdots & \ddots & \vdots & \ddots & \vdots \\
0 & \ldots & 1 & \ldots & 0
\end{array}\right]
$$

The matrix of unknown parameters $\xi$ can be written as

$$
\xi=\left[\begin{array}{ccc}
\xi_{11} & \ldots & \xi_{1 p} \\
\ldots & \ldots & \ldots \\
\xi_{k 1} & \ldots & \xi_{k p}
\end{array}\right]
$$

where $\xi_{j h}(j=1,2, \ldots, k, h=1,2, \ldots, p)$ is as above.

Application of longitudinal analysis makes it possible to assess differences between average increments of trees depending on environmental conditions as well as time. Three hypothesis are verified here:
1) $\quad \mathrm{H}_{01}:\left[\begin{array}{c}\xi_{11}-\xi_{12} \\ \ldots \\ \xi_{1 p-1}-\xi_{1 p}\end{array}\right]=\ldots=\left[\begin{array}{c}\xi_{k 1}-\xi_{k 2} \\ \ldots \\ \xi_{k p-1}-\xi_{k p}\end{array}\right]$,
2) $\quad \mathrm{H}_{02}: \sum_{h=1}^{p} \xi_{1 h}=\ldots=\sum_{h=1}^{p} \xi_{k h}$, 
3) $\quad \mathrm{H}_{03}: \sum_{j=1}^{k} \xi_{j 1}=\ldots=\sum_{j=1}^{k} \xi_{j p}$.

In (2) it is examined whether the differences between a consecutive time point are the same for all of a group. This means that the parallelism of profiles is checked. In the figure 1 the profile (mean heights of trees at each time point, i.e. year) are given for all generated tree heights grouped in four Kraft classes.

The hypothesis (2) can be written in matrix form as $\mathbf{C} \boldsymbol{\xi} \mathbf{M}$, where the $(k-1) \times k$ matrix $\mathbf{C}$ is the matrix of contrasts for groups

$$
\mathbf{C}=\left[\begin{array}{rrrrr}
1 & -1 & 0 & \ldots & 0 \\
0 & 1 & -1 & \ldots & 0 \\
\vdots & \vdots & \vdots & \ddots & \vdots \\
0 & 0 & 0 & \ldots & -1
\end{array}\right]
$$

and the $p \times(p-1)$ matrix $\mathbf{M}$ is the matrix of contrasts for time points, namely

$$
\mathbf{M}=\left[\begin{array}{rrrr}
1 & 0 & \cdots & 0 \\
-1 & 1 & \cdots & 0 \\
0 & -1 & \cdots & 0 \\
\vdots & \vdots & \ddots & 0 \\
0 & 0 & \cdots & -1
\end{array}\right]
$$

In order to verify (2), one-factor multivariate analysis of variance is used for $p$-1 differences of expectations for consecutive variables (time points) in each of the groups. The largest root of Roy $\theta=\lambda_{1} /\left(1-\lambda_{1}\right)$ (Krzyśko, 2000 p. 194), based on the largest eigenvalue $\lambda_{1}$ of matrix $\mathbf{H E}^{-1}$, whose dimensions are $(p-1) \times(p-1)$, must be determined for this purpose, where

$$
\begin{aligned}
& \mathbf{H}=\mathbf{M}^{\prime}\left(\mathbf{Y}^{\prime} \mathbf{X}\left(\mathbf{X}^{\prime} \mathbf{X}\right)^{-1} \mathbf{C}^{\prime}\right)\left[\mathbf{C}\left(\mathbf{X}^{\prime} \mathbf{X}\right)^{-1} \mathbf{C}^{\prime}\right]^{-1}\left(\mathbf{C}\left(\mathbf{X}^{\prime} \mathbf{X}\right)^{-1} \mathbf{X}^{\prime} \mathbf{Y}\right) \mathbf{M} \\
& \mathbf{E}=\mathbf{M}^{\prime}\left\{\mathbf{Y}^{\prime}\left[\mathbf{I}-\mathbf{X}\left(\mathbf{X}^{\prime} \mathbf{X}\right)^{-1} \mathbf{X}^{\prime}\right] \mathbf{Y}\right\} \mathbf{M}
\end{aligned}
$$

The hypothesis (2) should be rejected if the calculated value of statistic $\theta$ exceeds the table value, where $S=\min (k-1, p-1), M=(|k-p|-1) / 2$ and $N=(n k-k-p) / 2($ Krzyśko, 2000 p. 330). 
If there is no reason to reject the hypothesis (2), the hypothesis (3) of the equality of group effects (equality of profile) should be verified. This hypothesis is checked by means of one-dimensional one-factor analysis of variance (Morrison, 1990). The matrix form of this hypothesis is $\mathbf{C} \boldsymbol{\xi} \mathbf{m}_{1}$, where $\mathbf{m}_{1}$ is the column vector composed of $p$ ones. Matrices $\mathbf{H}$ and $\mathbf{E}$ are calculated by the formulas (5), substituting vector $\mathbf{m}_{1}$ in the place of matrix $\mathbf{M}$ (in this case matrices $\mathbf{H}$ and $\mathbf{E}$ have dimension $(1 \times 1))$. Similarly as in the case of hypothesis (2) the only nonzero eigenvalue of the matrix $\mathbf{H E}^{-1}$ can be calculated and compared with the critical value of the beta distribution. Because in this case $S=\min (1, p-1)=1$, it should be better to verify this hypothesis with analysis of variance conducted for the variables that are the sum of observations $y_{i j .}=\sum_{h=1}^{p} y_{i j h}$. If the hypothesis is rejected then in order to find significant differences among groups the Scheffe intervals can be used. The critical value for construction of Scheffe intervals are determined by the following formula

$$
t_{c t i t, g}=\sqrt{(k-1) F_{\alpha ; k-1, n k-k}},
$$

and the test statistics for the comparison of consecutive groups ( $l$ with $l+1$, where $l=1, \ldots, k-1$ ) take the form

$$
t_{l, g}=\mathbf{c}_{l} \overline{\mathbf{y}}_{k} \sqrt{\frac{n}{2 M S E}},
$$

where $M S E$ is the mean square for the error, $\mathbf{c}_{l}$ is the $l$ th row of matrix $\mathbf{C}$, and $\overline{\mathbf{y}}_{k}$ is a vector whose elements are the means $\bar{y}_{. j .}=(n p)^{-1} \sum_{i=1}^{n} \sum_{h=1}^{p} y_{i j h}$ $(j=1, \ldots, k)$.

Next the hypothesis (4) of equality of expectations $p$ of time points is verified. The matrix form for this hypothesis is $\mathbf{c}_{1} \boldsymbol{\xi} \mathbf{M}$, where $\mathbf{c}_{1}$ is the vector composed of $k$ ones. To comment on the equality of these expectations the $T^{2}$ Hotelling statistic is used. Because $S=\min (k-1,1)=1$, matrix $\mathbf{H E}^{-1}$ has only one nonzero eigenvalue, on which the test statistic is based:

$$
T^{2}=[(n k-k) n k] \overline{\mathbf{y}}_{p}{ }^{\prime} \mathbf{M} \mathbf{E}^{-1} \mathbf{M}^{\prime} \overline{\mathbf{y}}_{p},
$$


where the elements of vector $\overline{\mathbf{y}}_{p}$ are the means $\bar{y}_{. . h}=(n k)^{-1} \sum_{i=1}^{n} \sum_{j=1}^{k} y_{i j h}$ $(h=1, \ldots, p)$. If the above hypothesis is rejected, then multiple comparisons of time point expectations are performed using modified Hotelling statistics (Morion, 1990 str. 208).

The critical value for simultaneous comparison of time points is

$$
t_{c r i t, t}=\sqrt{\frac{(p-1)(n k-k)}{n k-k-p+2} F_{\alpha ; p-1, n k-k-p+2}},
$$

and the statistic for the $l$ th $(l=1, \ldots, p-1)$ comparison (contrast $\left.\bar{y}_{l}-\bar{y}_{l+1}\right)$ is as follows

$$
t_{l, t}=\mid \mathbf{m}_{l}^{\prime} \overline{\mathbf{y}}_{p} \sqrt{\frac{n k}{\mathbf{m}_{l}^{\prime} \text { Var } \mathbf{m}_{l}}},,
$$

where $\mathbf{m}_{l}$ is the $l$ th column of matrix $\mathbf{M}, \overline{\mathbf{y}}_{p}$ is as above and Var is the covariance matrix among time points.

If there is no interaction between time points and groups, then hypothesis (2) is rejected. At least for one group the dependence of height increments on time is different than for the remaining ones. Moreover, the rejection of hypothesis $\mathrm{H}_{01}$ automatically causes the rejection of hypotheses $\mathrm{H}_{02}$ and $\mathrm{H}_{03}$. Then, using for example simultaneous Roy confidence intervals (also used for multiple comparison in multivariate analysis of variance), the groups that influenced the rejection of (2) should be found (Morison, 1990 p. 302).

If hypothesis (2) is rejected, then the equality of groups is checked separately for each time point using $p$ one-dimensional analysis of variance and multiple comparison for groups. In the case of rejection of the hypothesis of equality of group effects (3), multiple comparisons should be performed using Scheffe confidence intervals. Similar procedures should be applied for examination of the equality of expectations calculated for consecutive time points separately for each group. In this case the one-sample $T_{j}^{2}=n \overline{\mathbf{y}}_{j}{ }^{\prime} \mathbf{M}\left(\mathbf{M}^{\prime} \operatorname{Var} \mathbf{M}\right) \mathbf{M}^{\prime} \overline{\mathbf{y}}_{j}$ statistic is used $(j=1, \ldots, k)$. In this formula $\overline{\mathbf{y}}_{j}$ is the vector of $p$ means $\bar{y}_{\cdot j h}=n^{-1} \sum_{i=1}^{n} y_{i j h}(h=1, \ldots, p)$ and $\operatorname{Var}$ is the 
covariance matrix among time points in the $j$ th group. The method of calculation of the above statistic and appropriate critical value $T_{c r i t}^{2}$ are given in the paper by Graczyk et al. (2010). The statistic for the $l$ th $(l=1, \ldots, p-1)$ comparison (contrast $\bar{y}_{. j l}-\bar{y}_{. j l+1}$ ) is as follows $T_{j l}=\left|\mathbf{m}_{l}^{\prime} \overline{\mathbf{y}} \sqrt{n / \mathbf{m}_{l}^{\prime} \operatorname{Var} \mathbf{m}_{l}}\right|$, where $\mathbf{m}_{l}$ is the $l$ th column of matrix $\mathbf{M}$.

Similarly, in the situation where the hypothesis $\mathrm{H}_{01}$ is not rejected but one of the hypotheses $\mathrm{H}_{02}$ and $\mathrm{H}_{03}$ (or both) is rejected, the significant differences among groups and time points are calculated. For this purpose Roy and Bose's (1953) intervals are used, in the same way as in the case of rejection of all three hypotheses. The method for determining intervals is given in the paper by Graczyk et al. (2010).

In the case of the discussed trial, in the model (1) we have $i=1,2, \ldots, 30$, $j=1,2, \ldots, 4, h=1,2, \ldots, 7$. The matrix $\Sigma$ is column full rank, $r(\Sigma)=7$.

The matrix of unknown parameters is:

$$
\xi=\left[\begin{array}{llll}
\xi_{11} & \xi_{12} & \cdots & \xi_{17} \\
\xi_{21} & \xi_{22} & \cdots & \xi_{27} \\
\xi_{31} & \xi_{32} & \cdots & \xi_{37} \\
\xi_{41} & \xi_{42} & \cdots & \xi_{47}
\end{array}\right] .
$$

In our trial the hypothesis (2) takes the form:

$$
\mathrm{H}_{01}:\left[\begin{array}{c}
\xi_{11}-\xi_{12} \\
\xi_{12}-\xi_{13} \\
\ldots \\
\xi_{16}-\xi_{17}
\end{array}\right]=\left[\begin{array}{c}
\xi_{21}-\xi_{22} \\
\xi_{22}-\xi_{23} \\
\ldots \\
\xi_{26}-\xi_{27}
\end{array}\right]=\left[\begin{array}{c}
\xi_{31}-\xi_{32} \\
\xi_{32}-\xi_{33} \\
\ldots \\
\xi_{36}-\xi_{37}
\end{array}\right]=\left[\begin{array}{c}
\xi_{41}-\xi_{42} \\
\xi_{42}-\xi_{43} \\
\ldots \\
\xi_{46}-\xi_{47}
\end{array}\right] \text {. }
$$

For example, vector $\left[\begin{array}{c}\xi_{11}-\xi_{12} \\ \xi_{12}-\xi_{13} \\ \ldots \\ \xi_{16}-\xi_{17}\end{array}\right]$ means the differences of height increments of trees at seven successive time points for the first social position group. 
The hypothesis (3) regarding equality of effects in each of the four groups is

$$
\mathrm{H}_{02}: \sum_{h=1}^{7} \xi_{1 h}=\sum_{h=1}^{7} \xi_{2 h}=\sum_{h=1}^{7} \xi_{3 h}=\sum_{h=1}^{7} \xi_{4 h} .
$$

$\sum_{h=1}^{7} \xi_{1 h}=\xi_{11}+\xi_{12}+\ldots+\xi_{17}$ means the sum of tree height increments at successive time points for the first group. Hypothesis (4) takes the form

$$
\mathrm{H}_{03}: \sum_{j=1}^{4} \xi_{j 1}=\sum_{j=1}^{4} \xi_{j 2}=\ldots=\sum_{j=1}^{4} \xi_{j 7},
$$

$\sum_{j=1}^{4} \xi_{j 1}=\xi_{11}+\xi_{21}+\xi_{31}+\xi_{41}$ means the sum of average increments of tree heights at the first time point for four groups.

\section{Results}

At the significance level $\alpha=0.05$ it was verified that the measurement error vectors were not correlated, i.e. they were independent. The correlation coefficients and their $p$-values are presented in Table 1 .

Table 1. Partial correlation coefficients and $p$-value of error vectors (the number

\begin{tabular}{|c|c|c|c|c|c|c|c|c|}
\hline & $\begin{array}{l}\text { Time } \\
\text { point }\end{array}$ & 1 & 2 & 3 & 4 & 5 & 6 & 7 \\
\hline \multirow{2}{*}{$\begin{array}{l}\text { Correlation coefficient } \\
\text { p-value }\end{array}$} & \multirow{2}{*}{1} & \multirow[t]{12}{*}{1} & -0.135 & 0.079 & 0.160 & 0.035 & 0.155 & 0.117 \\
\hline & & & 0.148 & 0.397 & 0.086 & 0.703 & 0.096 & 0.211 \\
\hline \multirow{2}{*}{$\begin{array}{l}\text { Correlation coefficient } \\
\text { p-value }\end{array}$} & \multirow{2}{*}{2} & & 1 & -0.004 & 0.033 & 0.080 & 0.230 & -0.252 \\
\hline & & & & 0.965 & 0.726 & 0.392 & 0.013 & 0.006 \\
\hline \multirow{2}{*}{$\begin{array}{c}\text { Correlation coefficient } \\
\text { p-value }\end{array}$} & \multirow{2}{*}{3} & & & 1 & 0.0642 & 0.061 & -0.167 & -0.052 \\
\hline & & & & & 0.492 & 0.513 & 0.073 & 0.581 \\
\hline \multirow{2}{*}{$\begin{array}{l}\text { Correlation coefficient } \\
\text { p-value }\end{array}$} & \multirow{2}{*}{4} & & & & 1 & -0.007 & 0.021 & 0.077 \\
\hline & & & & & & 0.938 & 0.825 & 0.411 \\
\hline \multirow{2}{*}{$\begin{array}{l}\text { Correlation coefficient } \\
\text { p-value }\end{array}$} & \multirow{2}{*}{5} & & & & & 1 & 0.051 & -0.083 \\
\hline & & & & & & & 0.587 & 0374 \\
\hline \multirow{2}{*}{$\begin{array}{c}\text { Correlation coefficient } \\
\text { p-value }\end{array}$} & \multirow[t]{2}{*}{6} & & & & & & 1 & -0.065 \\
\hline & & & & & & & & 0.482 \\
\hline
\end{tabular}
of degrees of freedom is 116)

Italics - significant coefficients at the level $\alpha=0.05$

Using the multivariate test of Royston (1983) it was verified, at the significance level $\alpha=0.05$, that the errors had a normal multivariate distribution with zero expectation and equal covariance matrices. The value of 
Royston's test statistic was $G^{*}=10.861$ and the critical table value was $\chi_{7}^{2}=14.067$.

For data related to the four Kraft classes a profile analysis was applied for model (1). The largest root of Roy $\theta$ based on the largest eigenvalue of matrix $\mathbf{H E}^{-1}$ was $\theta=0.859$. The critical table value (for parameters $S=3, M=1$, $N=54.5$ ) was $\theta_{t a b}=0.167$ (Krzyśko, 2000 p. 331). Thus the hypothesis (2) of profile equality was rejected at the significance level $\alpha=0.05$. Simultaneously the hypotheses of equality of profiles (3) and of time point effects (4) were rejected.

In order to find which tree groups (Kraft classes) influenced the lack of profile equality we used confidence intervals (Morion, 1900 paragraph 5.5). The critical value for differences between classes was $t_{\text {crit }, g}=2.8370$. The values of test statistics were respectively 3.859 for the comparison of class I and II, 5.450 for the comparison of class II and III and 4.134 for the comparison of class III and IV. The results of comparisons of groups are presented in Table 2. Kraft classes formed pairs of homogeneous groups.

Table 2. Multivariate multiple comparisons for groups of trees - Kraft classes.

The hypothesis (3) is rejected

\begin{tabular}{ccccc}
\hline Group - Kraft class & I & II & III & IV \\
\hline Homogeneous & a & a & & \\
group & & b & b & \\
& & & $c$ & $c$ \\
\hline
\end{tabular}

As the hypothesis (4) was rejected it means that time points (years) differed significantly. The critical value for their contrasts was $t_{c r i t, t}=3.7695$, and the statistic values for consecutive contrasts are given in Table 3 .

Since all the hypotheses were rejected, separate analyses should be carried out for each class and each year. 
Table 3. Comparisons (contrasts) of consecutive measurement years - time points $t_{\text {crit }, t}=3.7695$. The hypothesis (4) is rejected

\begin{tabular}{c|cccccc} 
contrasts & $1-2$ & $2-3$ & $3-4$ & $4-5$ & $5-6$ & $6-7$ \\
\hline$t_{l}$ & 7.688 & 6.261 & 9.820 & 6.973 & 7.914 & 4.775
\end{tabular}

In the first step there were performed $p=7$ one-dimensional analyses of variance, separately for each measurement year, in order to verify equality of groups. The results are presented in Table 4. In the third, fourth, sixth and seventh measurement years all groups significantly differed from each other, however in the first and second year classes I and II as well as III and IV constituted homogeneous groups, and in the fifth year only classes I and II.

Table 4. The homogeneous groups for Kraft classes in consecutive measurement years - time points

\begin{tabular}{|c|c|c|c|c|c|c|c|c|c|c|c|c|}
\hline & \multicolumn{10}{|c|}{ Time point of height measurements } \\
\hline & & & 1 & & 2 & & & 4 & 5 & & & l \\
\hline 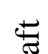 & & I & $\mathrm{a}$ & & $\mathrm{a}$ & & $\mathrm{a}$ & $\mathrm{a}$ & a & & $\mathrm{a}$ & $\mathrm{a}$ \\
\hline 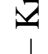 & 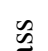 & II & $\mathrm{a}$ & & $\mathrm{a}$ & & $\mathrm{b}$ & b & $\mathrm{a}$ & & b & $\mathrm{b}$ \\
\hline 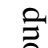 & $\overline{0}$ & III & & $\rho$ & & & $\mathrm{c}$ & $\mathrm{c}$ & & & c & $\mathrm{c}$ \\
\hline ப் & & IV & & b & & 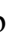 & d & d & & c & $\mathrm{d}$ & $\mathrm{d}$ \\
\hline
\end{tabular}

In the second step the profile analysis was performed separately for each of the four Kraft classes. The statistics $T_{j}^{2}$ for all classes exceeded the critical value (the boundary significance level $-p$-value - was in each case smaller than 0.0001), therefore for each group the null hypothesis of lack of differences between years was rejected. The statistic values $T_{j}^{2}$ in the groups were respectively: class I 2736.25, class II 547.73, class III 3962.83, class IV 687.47, and the critical value was $T_{\text {ctit }}^{2}=18.184$, for $\alpha=0.05$ and $p-1=6$, $n-p+1=24$ degrees of freedom for the numerator and denominator respectively.

In order to evaluate which years differed significantly in particular groups, Scheffe intervals were defined for comparisons between consecutive measurement years (for particular contrasts). In Table 5, instead of intervals, 
there are given the values of $T_{j l}$ statistics together with the corresponding critical value $T_{\text {crit }}=\sqrt{T_{\text {crit }}^{2}}$. In group I (Kraft class) year 1 and 2 as well as 4 and 5 did not differ significantly, while in group II there were no notable differences between years - all contrasts were insignificant. In class III there were no significant differences between years 3 and 4 as well as 6 and 7, while in class IV significant differences were found only for the contrast between years 3 and 4.

Table 5. Statistic values for comparisons (contrasts) of consecutive measurement years in groups - Kraft classes. $T_{\text {crit }}=4.264313$

\begin{tabular}{ccccc}
\hline \multirow{2}{*}{ Points of time } & \multicolumn{4}{c}{ Group - Kraft class } \\
\cline { 2 - 5 } & I & II & III & IV \\
\hline $1-2$ & 4.24 & 3.58 & 4.56 & 2.91 \\
$2-3$ & 5.80 & 1.51 & 5.47 & 1.91 \\
$3-4$ & 8.07 & 3.91 & 3.05 & 6.26 \\
$4-5$ & 2.00 & 4.08 & 5.69 & 2.57 \\
$5-6$ & 8.11 & 2.46 & 5.20 & 1.68 \\
$6-7$ & 5.57 & 1.38 & 2.74 & 2.32 \\
\hline
\end{tabular}

\section{Conclusions}

The analysis leads to the conclusion that tree height increments were not parallel in all groups, i.e. the Kraft classes differed regarding the increments of the studied characteristics. Moreover, both Kraft classes and consecutive measurement years showed that they formed homogeneous groups joining two neighboring classes in pairs, which is a relatively natural conclusion and proves that the method applied for the classification of trees was adequate. All contrasts between consecutive years were significant. Therefore it can be concluded that weather conditions significantly influenced tree height increments.

The comparisons between groups performed separately for each measurement year showed that in four out of seven years no homogeneous 
groups were found and each Kraft class differed from the others significantly. In the two first measurement years there were found two homogeneous groups: one consisting of classes I and II and the other of classes III and IV. Therefore it may be stated that the division of trees into groups was formed in the initial period (when the trees were 18-19 years old) and in the later period this division was sustained. It must be added, however, that this does not provide a complete picture of changes between the groups, since in the production forests dried trees are immediately removed and there is no data regarding such units.

Due to the fact that all consecutive measurement years differed from each other significantly regarding the studied tree characteristics, the comparisons between years were conducted separately for each Kraft class. It appeared that in group II and IV most years (compared in pairs) did not differ significantly. However, in the other groups the situation was the opposite. The results may be partly explained by the natural tendency of dominating trees to grow faster and of the weaker trees (from class IV) to have smaller and smaller height increments, consequently leading to their becoming dry. However, the height increment of trees is influenced not only by their social position, but also by their age, the weather conditions and many other factors.

\section{REFERENCES}

Assmann E. (1968): Nauka o produkcyjności lasu [Forest yield science]. PWRiL, Warszawa. [in Polish].

Graczyk M., Kaźmierczak K., Zawieja B. (2010): The longitudinal analysis applied to the analysis of height increment of pine stands. Biometrical Letters 47(2): 199-128.

Kaźmierczak K., Zawieja B. (2008): An attempt to assess the correlation between lengthwise growth of the main shoot in 24-year old Scots pines (Pinus sylvestris $\mathbf{L}$ ) on the growth of lateral branches. Biometrical Letters 45(1): 81-92.

Krzyśko M. (2000): Wielowymiarowa analiza statystyczna. [Multivariate statistical analysis] Wydawnictwo UAM Poznań. [in Polish].

Lemke J. (1971): Przydatność niektórych cech dendrometrycznych do wyboru przyrostowych drzew próbnych w drzewostanach sosnowych [Suitability of certain dendrometric traits in the selection of increment mean sample trees in pine stands]. Pr. Kom. Nauk Roln. Nauk Leśn. PTPN 32: 57-72. [in Polish].

Morison D.F. (1990): Wielowymiarowa Analiza Statystyczna [Multivariate statistical methods]. PWN, Warszawa. [in Polish]. 
Roy S.N., Bose R.C. (1953): Simultaneous confidence intervals estimation. Annals of Mathematical Statistics 24: $513-538$.

Royston J.P. (1983): Some techniques for assessing multivariate normality based on the Shapiro-Wilk W. Applied Statistics 32: 121-133. 